\title{
Reduced Order Unsteady Aerodynamic Model of a Rigid Aerofoil in Gust Encounters
}

\author{
Qiang Zhou ${ }^{\mathrm{a}}$, Gang Chen ${ }^{\mathrm{a}, *}$, Andrea Da Ronch ${ }^{\mathrm{b}, * *}$, Yueming $\mathrm{Li}^{\mathrm{a}}$ \\ ${ }^{a}$ State Key Laboratory for Strength and Vibration of Mechanical Structures, and \\ Shannxi Key Laboratory for Environment and Control of Flight Vehicle, \\ Xi'an Jiaotong University, Xi'an, China \\ ${ }^{b}$ Faculty of Engineering and the Environment, \\ University of Southampton, Southampton, SO17 1BJ, U.K.
}

\begin{abstract}
Predicting gust loads using computational fluid dynamics is prohibitively expensive and unrealistic for parametric searches. This work presents the development, implementation, and demonstration of a reduced order model which balances accuracy and speed. The model builds on a proper orthogonal decomposition representation of the linearised time-domain equations and achieves a further reduction in size through a balanced truncation. The novelty of the work lies in the mechanism to introduce any arbitrary gust shape within the reduced order model framework. The methodology combines an analytical formulation, loosely based on the Küssner function, and a numerical approach to identify, or optimise, the unknown parameters of the analytical ansatz. A model problem is investigated for various gust shapes for incompressible and transonic flows. It is found that: (i) the generation of the reduced order model is equivalent to about two steady-state analyses;
\end{abstract}

*Second corresponding author. Email: aachengang@mail.xjtu.edu.cn

**First corresponding author. Email: A.Da-Ronch@soton.ac.uk 
(ii) the predictions of the reduced order model are in good to excellent agreement with the reference solution; and (iii) the reduced order model achieves a consistent speed-up of about 300 times compared to time integrating the original equations. The reduced order model is parametric with respect to the gust disturbance, and may be employed for the worst-case-gust search without extra costs.

Keywords: gust loads, computational fluid dynamics, reduced order model, unsteady aerodynamics

\section{Nomenclature}

$a$

$\boldsymbol{A}(\alpha)$

$\boldsymbol{A}, \boldsymbol{B}, \boldsymbol{C}$

$b$

$c$

$\boldsymbol{F}$

$\boldsymbol{H}$

$H_{g}$

$m$

$M$

$n$

$r$

$s$

$t^{*}$

$t_{f}^{*}$ speed of sound

volume of fluid cell

matrices of the linearised time domain equations

reference semi-chord

parameter to be identified in the reduced model

inviscid flux vector

Jacobian matrix of $\boldsymbol{F}$ with respect to $\boldsymbol{w}$

non-dimensional gust wavelength normalised by the reference semi-chord

dimension of the full order model

freestream Mach number

dimension of the reduced order model

dimension of the reduced order model after balanced trunction

non-dimensional time based on reference semi-chord

non-dimensional time based on reference chord

non-dimensional final time based on reference chord 
$U$

$u_{g}, w_{g}$

$w_{g 0}$

$\boldsymbol{w}$

$\boldsymbol{x}$

2 Greek

$\alpha$

$\beta$

$\Delta$

$\varepsilon_{1}, \varepsilon_{2}, \Phi_{1}, \Phi_{2}$

$\varepsilon_{3}, \varepsilon_{4}, \Psi_{1}, \Psi_{2}$

$\lambda$

$\Phi, \Psi$

3

Abbreviations

BT

CFD

$\operatorname{DoF}(\mathrm{s})$

POD

ROM freestream speed

horizontal and vertical components of the gust velocity

maximum intensity of vertical component of the gust velocity vector of conservative flow variables

state vector of the linearised time domain equations

angle of attack

parameter to be identified in the reduced model

perturbation variable

coefficients in the Wagner approximation

coefficients in the Küssner approximation

Lagrange multiplier, and advance ratio

subspaces in the proper orthogonal decomposition

balance trunction

computational fluid dynamics

degree(s) of freedom

proper orthogonal decomposition

reduced order model 
4

5

\section{Introduction}

Specific aircraft performance parameters are monitored and stored by the digital flight data recorder. These data include, among more than 900 parameters, the aircraft body rates and attitude, speed, altitude, and load factors, which can be used to reconstruct the properties of any turbulence encountered by in-flight transport aircraft (1). The reasons this dataset is valuable to aircraft manufacturers and airliners are two-fold. First, with an adequate dynamic model of the aircraft, loads on any point of the airframe can be reconstructed, providing indications on whether design target loads were reached during operation. The second reason is that knowledge of the actual loads experienced by the aircraft during flight allows optimising the time interval of inspections and grounding, and targeting specific structural components of the airframe.

The ability to perform the above mentioned tasks relies on the availability of a model of the aircraft dynamics, which includes the effects of atmospheric turbulence and gusts on the rigid and flexible body dynamics. To make progress in this direction, the work carried out in this paper presents the implementation of a rapid gust loads analysis capability based on computational fluid dynamics (CFD) and its application to arbitrary-shaped gust encounters.

For an incompressible, irrotational and two-dimensional flow around a flat plate, Theodorsen (2) provides an analytical formulation of the unsteady aerodynamic loads. The Wagner function describes the indicial built-up of the circulatory part of the lift, including the influence of the shed wake. Although known exactly in terms of Bessel functions, the Wagner function 
is practically expressed as an exponential approximation

$$
\phi(s)=1-\Phi_{1} e^{-\varepsilon_{1} s}-\Phi_{2} e^{-\varepsilon_{2} s}
$$

with the coefficients $\Phi_{1}=0.165, \Phi_{2}=0.335, \varepsilon_{1}=0.0455$, and $\varepsilon_{2}=0.3$ from R. T. Jones (3). It is interesting to observe that at the initial time $\phi(0)=0$. The Küssner function, on the other hand, gives the lift built-up for the penetration into a sharp-edged gust. Like the Wagner function, it is known exactly in terms of Bessel functions, but an exponential approximation is generally employed for calculations

$$
\psi(s)=1-\Psi_{1} e^{-\varepsilon_{3} s}-\Psi_{2} e^{-\varepsilon_{4} s}
$$

where the coefficients $\Psi_{1}=0.5792, \Psi_{2}=0.4208, \varepsilon_{3}=0.1393$, and $\varepsilon_{4}=1.802$ are taken from Ref. (4). Note that $\psi(0)=0$, and that the non-dimensional time used in the analytical formulation, $s=t U_{\infty} / b$, is normalised by the reference semi-chord, $b$. This is in contrast to the convention generally adopted in CFD, where the non-dimensional time is defined as $t^{*}=t U_{\infty} / 2 b$. For a moving sharp-edged gust with variable propagation speed, in which the gust front moves towards or away from the aerofoil, an analytical formulation is given in Ref. (5). Exact analytical expressions of the indicial response to a step change in angle of attack, a step change in pitch rate, and for the penetration into a sharp-edged gust in subsonic compressible flow were obtained by Lomax (6). For a compressible flow, there are no exact closed-form analytical solutions for all times. By use of linear piston theory (7), the initial values of the indicial response can be obtained, and the final values are given by a steady-state method. 


\section{Gust Analysis using Computational Fluid Dynamics}

Indicial aerodynamics is a powerful mathematical tool based upon the assumption of linearity. Once the indicial response is known, the system response to any arbitrary input signal can be calculated by the convolution or Duhamel's integral. The predictive capabilities of indicial aerodynamics are, however, restricted to conditions where the principle of superposition is valid. To overcome this issue, researchers investigated a number of alternatives. The first attempts to directly determine the indicial response by CFD date back to 1990s (8). This approach has received widespread use but presents a number of challenges (9; 10). Considering that an unsteady time-domain CFD analysis is needed to compute the indicial response, large computational resources are expected making any iterative process impractical. A second major difficulty is that an unsteady CFD analysis is sensitive to the spatial and temporal resolutions, grid quality, and the dissipation of the numerical scheme. More recently, a combined analytical-numerical approach was proposed in Ref. (11), whereby the predictive capabilities of CFD are employed to revise and extend the (classic) analytical formulations. In that work, aerodynamic indicial functions for a compressible subsonic flow were obtained by modifying those for an incompressible flow using the PrandtlGlauert transformation for the circulatory part of the lift, and using the piston theory for the non-circulatory part. The coefficients of the revised formulation were then fine-tuned with the CFD response for aerofoils and elliptic wings. Reference (12) extended that approach to applications on trapezoidal wings for different aspect ratios and wing sweep angles, for Mach numbers between 0.3 and 0.7 . A number of assessments were carried out, 
revealing the computational efficiency and general predictive capability of the new analytical formulation.

High-fidelity models based on CFD are needed for transonic as well as massively separated flows. The cost of time-domain calculations makes the routine use of CFD in gust response analysis unrealistic, and reduced order models (ROMs) have been used as a cheaper alternative. For example, Ref. (13) investigated the identification of four ROMs for the response of a rigid three-dimensional wing to a travelling gust and its coupling with the aeroelastic equations of motion. The ROMs included a convolution model identified from a CFD-based sharp-edged gust response, a parametric autoregressive moving average and state space model, and a frequency-response model. The training data for the latter three ROMs were for a random gust excitation with Gaussian distribution. The models were found computationally efficient and reproduced the CFD response accurately in the subsonic flow regime. Then, Ref. (14) generated a ROM of the unsteady aerodynamics based on the autoregressive with exogenous input model. The test case was for the BAH wing (15) using one rigid body mode and five flexible modes. After the ROM was generated, which is the most expensive step, good predictions for a single flight point were reported. Reference (16) presented the development of a CFD-based gust model for the Boeing truss braced wing aircraft. The ROM combines the convolution of a sharp-edged gust with a model constructed from the proper orthogonal decomposition (POD) of the covariance matrix of the sharp-edged gust unsteady pressure coefficients. The ROM was found in good agreement with the CFD solution for a "one-minus-cosine" gust, and was then employed to compute at no 
extra costs a familiy of "one-minus-cosine" gust responses.

Further applications of the POD method include the generation of CFDbased ROMs for control design synthesis. Reference (17) performed active flutter suppression and control design for gust loads alleviation using a POD ROM, whereas a further reduction in the ROM size using a balanced truncation (BT) method was demonstrated in Ref. (18).

System identification models, like those reviewed above and the references therein, suffer from the usual limitations related to the necessity for a set of training data closely related to the final application cases, and the difficulty of accounting for the non-linearity in the reduced model. A systematic and cost effective approach to developing ROMs capable of describing both linear and nonlinear effects for a range of cases based on limited development cost has, to date, proved elusive. A novel approach to the reduction of nonlinear models for gust loads prediction was first introduced in Ref. (19). The method uses information on the eigenspectrum of the coupled system Jacobian matrix and projects the full order model through a series expansion onto a small basis of eigenvectors which is capable of representing the full order model dynamics. Linear and nonlinear ROMs derived from linear unsteady aerodynamics/CFD and linear/nonlinear structural models were generated. The application to the Goland wing was documented in Ref. (20), and that to a complete aircraft configuration in Ref. (21). The method has several strengths: (i) it exploits information from the stability (flutter) calculation for the development of a ROM for dynamic response analyses; (ii) linear or nonlinear reduced models can be developed within the same framework; and (iii) the reduced model can be parametrised to avoid ROM regeneration. 
The main contribution of this paper is the introduction of gust terms in a CFD-based ROM which allows arbitrary-shaped gust encounters to be analysed. While limited to a thorough investigation of the gust loads around a rigid aerofoil, the methods were demonstrated adequate for predictions around a three-dimensional flexible configuration (22). This extension will be reported in a future manuscript.

The paper continues in Section 3 with a description of the CFD solver and the ROM generation. Section 4 provides a thorough validation of CFD predictions for gust loads with available data in the literature. Then, Section 5 compares the gust loads computed using the ROM with those using the CFD solver, evaluating the accuracy and the computational costs. Finally, conclusions are given in Section 6 .

\section{Formulation}

\subsection{Computational Fluid Dynamics Solver}

The research CFD solver used herein is a cell-centred finite volume code solving the unsteady Reynolds-averaged Navier-Stokes equations (URANS) on block structured grids. The spatial discretisation is based on the second order Van Leer scheme (23). For time marching the governing equations, the Lower Upper Symmetric Gauss-Seidel (LU-SGS) method is used. The accuracy of the baseline CFD code, both steady and unsteady, has been previously validated and more information about the numerical details may be found in Refs. $(24 ; 25)$. Results in this paper are for the Euler equations. This is a reasonable assumption for the high Reynolds number of the conditions considered, where viscous effects can be neglected. 
The discrete system of non-linear equations in time-domain using an Arbitrary Lagrangian-Eulerian method is

$$
\frac{d(\boldsymbol{A}(\alpha) \boldsymbol{w})}{d t^{*}}+\boldsymbol{F}(\boldsymbol{w}, \alpha)=\mathbf{0}
$$

where symbols are defined in the Nomenclature. The time domain equations depend generally on the motion of the aircraft. In this work, the parameter $\alpha\left(t^{*}\right)$ is taken to be the pitch angle of the aerofoil relative to the direction of the freestream speed, but the concept is easily generalised to arbitrary motions (22).

\subsubsection{Gust Treatment}

A methodology for CFD-based gust analysis was introduced in Ref. (8) and later practised in Ref. (26). The approach, referred to as the field velocity approach, is also exploited in this work. The gust is introduced into the CFD solver by modification of the velocity of grid points during the unsteady motion of the aircraft. An advantage of the field velocity approach is that it overcomes the problems associated with the numerical dissipation of the disturbance, and no requirements on the spatial discretisation exist. A disadvantage is that the gust is assumed frozen, and the influence of the structural response on the gust is neglected. This assumption looses validity when second order effects, which occur in extreme flight conditions at the edges of the envelope, become important. The approach, however, has received widespread use because of the lack of alternative methods.

\subsection{Reduced Order Model}

The approach here presented to the reduction of a large non-linear CFD model for gust analysis consists of three steps: (i) linearisation of the gov- 
erning equations around a non-linear steady-state flow condition, discussed in Section 3.2.1: (ii) generation of a POD basis for the projection of the linearised flow equations, overviewed in Section 3.2.2 and (iii) identification of the optimal coefficients that are used to introduce gust perturbations within the ROM framework, discussed in Section 3.2 .3 .

\subsubsection{Linearised Time Domain Equations}

The linearised time domain equations are obtained by linearising Eq. (3) around a non-linear steady-state solution, $\left(\boldsymbol{w}_{0}, \alpha_{0}\right)$. Assuming an unsteady motion with a small amplitude, the unsteady terms can be expressed as a superposition of a steady mean state and a perturbation

$$
\begin{aligned}
& \boldsymbol{w}\left(t^{*}\right) \approx \boldsymbol{w}_{0}+\Delta \boldsymbol{w}\left(t^{*}\right), \alpha\left(t^{*}\right) \approx \alpha_{0}+\Delta \alpha\left(t^{*}\right) \\
& \|\Delta \alpha\| \ll\left\|\alpha_{0}\right\| \\
& \dot{\alpha}\left(t^{*}\right) \approx \Delta \dot{\alpha}\left(t^{*}\right)
\end{aligned}
$$

When linearizing about the (non-linear) steady mean state, Eq. (3) results in the following system of equations

$$
\boldsymbol{A}_{0} \Delta \dot{\boldsymbol{w}}+\boldsymbol{H} \Delta \boldsymbol{w}+\boldsymbol{G} \Delta \alpha+(\boldsymbol{E}+\boldsymbol{L}) \Delta \dot{\alpha}=\mathbf{0}
$$

where

$$
\begin{aligned}
\boldsymbol{A}_{0} & =\boldsymbol{A}\left(\alpha_{0}\right) \\
\boldsymbol{H} & =\frac{\partial \boldsymbol{F}}{\partial \boldsymbol{w}}\left(\boldsymbol{w}_{0}, \alpha_{0}\right) \\
\boldsymbol{G} & =\frac{\partial \boldsymbol{F}}{\partial \alpha}\left(\boldsymbol{w}_{0}, \alpha_{0}\right) \\
\boldsymbol{E} & =\frac{\partial \boldsymbol{A}}{\partial \alpha}\left(\alpha_{0}\right) \boldsymbol{w}_{0} \\
\boldsymbol{L} & =\frac{\partial \boldsymbol{F}}{\partial \dot{\alpha}}\left(\boldsymbol{w}_{0}, \alpha_{0}\right)
\end{aligned}
$$


The terms $\boldsymbol{G}, \boldsymbol{E}$ and $\boldsymbol{L}$ are calculated using a first order finite difference. Only the semi-discrete flux Jacobian matrix $\boldsymbol{H}$ requires special attention, and more details may be found in Ref. (27). Equation (5) is easily recast in a classic state space form

$$
\left\{\begin{array}{l}
\dot{x}=A x+B u \\
y=C x
\end{array}\right.
$$

where $\boldsymbol{x}=\Delta \boldsymbol{w}, \boldsymbol{A}=-\boldsymbol{A}_{0}^{-1} \boldsymbol{H}, \boldsymbol{B}=-\boldsymbol{A}_{0}^{-1}[\boldsymbol{G}, \boldsymbol{E}+\boldsymbol{L}], \boldsymbol{u}=[\Delta \alpha, \Delta \dot{\alpha}]^{T}$, $\boldsymbol{y}=\Delta \boldsymbol{f}^{\text {ext }}$, and $\boldsymbol{C}=\partial \boldsymbol{f}^{\text {ext }} / \partial \boldsymbol{w}\left(\boldsymbol{w}_{0}, \alpha_{0}\right)$. The second equation in Eq. (7) relates the flow solution to the aerodynamic quantities of interest (pressure coefficient, generalised forces and moments, etc.).

It is not uncommon for a CFD model to contain several million degrees of freedom. Whereas Eq. (7) has a lower degree of complexity than the nonlinear counterpart in Eq. (3), it retains the same size of the original problem. The POD technique, detailed in the next section, is employed to achieve a significant reduction on the size of the model by projecting the linearised time domain equations onto a small basis of POD modes.

\subsubsection{Proper Orthogonal Decomposition}

The POD technique is used to extract a small basis of modes that represent the dynamics of the original model, Eq. (7). The first step is to create a set of snapshots of the full order model, $\boldsymbol{X}=\left[\boldsymbol{x}^{1}, \ldots, \boldsymbol{x}^{m}\right] \in \mathbb{R}^{n \times m}$ where $m$ indicates the number of snapshots. Here, $\boldsymbol{x}^{k}$ represents the $k$-th snapshot spanning the $n$-dimensional space. For an arbitrarily chosen subspace, $\mathbf{\Phi}$, that satisfies $\boldsymbol{\Phi}^{T} \boldsymbol{\Phi}=\boldsymbol{I}$, the POD technique searches an $m$-dimensional 
proper orthogonal subspace, $\Psi \in \mathbb{R}^{n \times m}$, that minimises the error

$$
G=\min _{\boldsymbol{\Phi}} \sum_{k=1}^{m}\left\|\boldsymbol{x}^{k}-\boldsymbol{\Phi} \boldsymbol{\Phi}^{T} \boldsymbol{x}^{k}\right\|=\sum_{k=1}^{m}\left\|\boldsymbol{x}^{k}-\boldsymbol{\Psi} \boldsymbol{\Psi}^{T} \boldsymbol{x}^{k}\right\|
$$

The above minimisation problem is equivalent to

$$
H=\max _{\boldsymbol{\Phi}} \sum_{k=1}^{m} \frac{\left\langle\left(\boldsymbol{x}^{k}, \boldsymbol{\Phi}\right)^{2}\right\rangle}{\|\boldsymbol{\Phi}\|^{2}}=\sum_{k=1}^{m} \frac{\left\langle\left(\boldsymbol{x}^{k}, \boldsymbol{\Psi}\right)^{2}\right\rangle}{\|\boldsymbol{\Psi}\|^{2}}
$$

where the symbol $\langle\cdot, \cdot\rangle$ indicates an averaging operation, and $(\cdot, \cdot)$ indicates an inner product operation. Since $\boldsymbol{\Phi}$ has to satisfy $\boldsymbol{\Phi}^{T} \boldsymbol{\Phi}=\boldsymbol{I}$, the problem is reformulated as a constrained optimisation with the Lagrange multiplier, $\lambda$

$$
J(\boldsymbol{\Phi})=\sum_{k=1}^{m}\left(\boldsymbol{x}^{k}, \boldsymbol{\Phi}\right)^{2}-\lambda\left(\|\boldsymbol{\Phi}\|^{2}-1\right)
$$

By taking the partial derivative of $J(\Phi)$ with respect to the unknown subspace, $\boldsymbol{\Phi}$

$$
\frac{d}{d \boldsymbol{\Phi}} J(\boldsymbol{\Phi})=2 \boldsymbol{X} \boldsymbol{X}^{T} \boldsymbol{\Phi}-2 \lambda \boldsymbol{\Phi}
$$

the solution that minimises Eq. (8) is also the solution of the following equation

$$
\left(\boldsymbol{X} \boldsymbol{X}^{T}-\lambda \boldsymbol{I}\right) \Psi=\mathbf{0}
$$

Equation (12) is a large dimensional eigenvalue problem for the POD kernel, $\boldsymbol{K}=\boldsymbol{X} \boldsymbol{X}^{T}$. To avoid the complexity to numerically solve a large eigenvalue problem (28), and by observing that the eigenvalues of $\boldsymbol{X} \boldsymbol{X}^{T}$ are the same as those of $\boldsymbol{X}^{T} \boldsymbol{X}$, the solution can be found from a smaller ( $m$-dimensional) eigenvalue problem

$$
\left\{\begin{array}{l}
\boldsymbol{X}^{H} \boldsymbol{X} \boldsymbol{V}=\boldsymbol{V} \boldsymbol{\Lambda} \\
\boldsymbol{\Psi}=\boldsymbol{X} \boldsymbol{V} \boldsymbol{\Lambda}^{-1 / 2}
\end{array}\right.
$$


where $\boldsymbol{\Psi}=\left[\boldsymbol{\Psi}_{1}, \ldots, \boldsymbol{\Psi}_{m}\right]$ and $\boldsymbol{\Lambda}=\operatorname{diag}\left(\lambda_{1}, \ldots, \lambda_{m}\right)$ is a diagonal matrix where the eigenvalues are sorted for decreasing values, $\lambda_{1} \geq \lambda_{2} \geq \ldots \geq \lambda_{m}$. As the singular values bring information on the energy contained in the system, the number of POD modes is often truncated to match, with the least number of modes, a desired energy level of the original model. Define the total energy level contained by a POD basis with $r$ modes as:

$$
\eta(r)=\frac{\sum_{i=1}^{r} \lambda_{i}}{\sum_{i=1}^{m} \lambda_{i}}
$$

In this work, the number of POD modes retained ensures capturing 99.99\%, or $\eta(r)=0.9999$, of the energy of the original full order model.

Once the POD basis is created by retaining $r$ modes, a transformation of coordinates between the full order model, $\boldsymbol{x}$, and the reduced order model, $\boldsymbol{x}_{r} \in \mathbb{R}^{r}$, is established

$$
\boldsymbol{x}=\boldsymbol{\Psi}_{r} \boldsymbol{x}_{r}
$$

Finally, the linearised time domain equations, Eq. (7), are projected onto the subspace $\boldsymbol{\Psi}_{r}=\left[\boldsymbol{\Psi}_{1}, \ldots, \boldsymbol{\Psi}_{r}\right] \in \mathbb{R}^{n \times r}$

$$
\left\{\begin{array}{l}
\dot{\boldsymbol{x}}_{r}=\boldsymbol{\Psi}_{r}^{T} \boldsymbol{A} \boldsymbol{\Psi}_{r} \boldsymbol{x}_{r}+\boldsymbol{\Psi}_{r}^{T} \boldsymbol{B} \boldsymbol{u} \\
\boldsymbol{y}=\boldsymbol{C} \boldsymbol{\Psi}_{r} \boldsymbol{x}_{r}
\end{array}\right.
$$

Equation (16) represents the dynamic equations of the ROM derived from the linearised time domain equations. The advantage of the reduced order model is that it is significantly smaller in size than Eq. (7). Disclosing results from Section 5, the size of the original model is $n \approx \mathcal{O}\left(10^{5}\right)$ whereas that of the ROM is $r \approx \mathcal{O}\left(10^{1}\right)$. 


\subsubsection{Gust Treatment in the Reduced Order Model}

To begin, the gust disturbance is introduced in the ROM equations through the term $\boldsymbol{u}=[\Delta \alpha, \Delta \dot{\alpha}]^{T}$. The methodology herein proposed builds on two requirements: speed and simplicity. The first consideration is to avoid degrading the computational performance of the ROM for gust analysis. For example, several methods proposed in Ref. (19) require manipulating large matrices, as large as the degrees of freedom of the CFD model, considerably slowing down the time integration of the ROM equations. The second consideration of simplicity is inspired by the Küssner function that gives the lift built-up for a sharp-edged gust (recall Eq. (2)).

The main contribution of this paper is the calculation of the gust terms in the ROM. We propose to model the angle of attack due to a moving sharp-edge gust of intensity $\hat{w}_{g 0}=w_{g 0} / U$ as:

$$
\alpha_{\mathrm{SE}}\left(t^{*}\right)=\hat{w}_{g 0} c\left(1-e^{-\beta t^{*}}\right) \quad \text { for } 0<t^{*}<\frac{H_{g}}{U_{\infty} \lambda^{-1}}
$$

where $c$ and $\beta$ are parameters to be identified. The similarity with the Küssner function is evident. One exponential is retained as it provides good approximations. More exponentials can be added to refine the approximation if needed, without affecting the convergence of the series. The advance ratio日 $\lambda$, is defined in the usual way as:

$$
\lambda=\frac{U}{U+u_{g}}=\frac{M}{M+u_{g} / a}
$$

The angle of attack for any arbitrary gust input is obtained using the con-

${ }^{1}$ Note that the Lagrange multiplier is commonly denoted in the literature by $\lambda$, as in Eq. (10). For the remainder of the work, $\lambda$ is used to indicate the advance ratio. 
volution integral:

$$
\alpha\left(t^{*}\right)=\int_{0}^{t^{*}} \frac{d \hat{w}_{g}(\tau)}{d \tau} \alpha_{\mathrm{SE}}\left(t^{*}-\tau\right) d \tau
$$

which is used to introduce the gust terms in the ROM equations (recall Eq. (16)).

Equation (17) contains two parameters, $c$ and $\beta$, that need to be identified once during the ROM generation. The parameter $c$ is identified from the relation

$$
c=\frac{C_{L}\left(t_{f}^{*}\right)}{C_{L}^{\mathrm{ROM}}\left(t_{f}^{*}, \beta=0, c=1\right)}
$$

where the steady-state response (at the final time $t_{f}^{*}=N \Delta t$ ) to a sharpedged gust computed using the original CFD equations, $C_{L}$, is compared with that computed using the ROM, $C_{L}^{\mathrm{ROM}}$. The parameter $\beta$ rules the transient response and is calculated from a minimisation problem carried out in time:

$$
f=\min _{\beta} \sum_{i=0}^{N}\left(C_{L_{i}}-C_{L_{i}}^{\mathrm{ROM}}(\beta)\right)^{2}
$$

\section{Validation Problems}

Numerical results are compared to exact closed-form expressions obtained for a flat plate (6). To reduce the effects of thickness, a NACA 0012 aerofoil was used. A preliminary study was made to test that solutions presented are independent of the grid used, see Fig. 1. Three sets of grids were generated. The two-dimensional domain extends fifty chords from the solid wall to the farfield. The coarsest grid had a total number of 7,220 cells with 130 nodes around the aerofoil. A medium grid consisted of 11,020 cells where 230 nodes 
were distributed on the aerofoil. The finest grid was obtained with a total of 14,820 cells with 330 nodes around the aerofoil. Boundary conditions on the aerofoil surface are set to inviscid wall, and those at the farfield set to Riemann farfield boundary conditions. The computational mesh for the solution of the Euler equations on the medium grid was found adequate, and results presented hereafter are for the medium grid shown in Fig. 1(b).

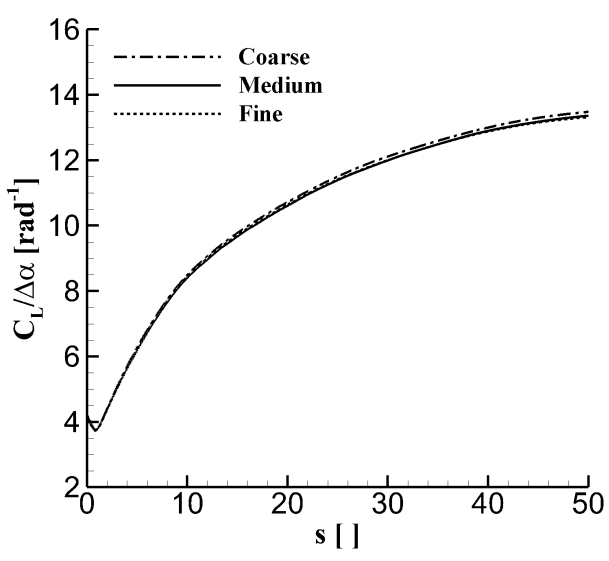

(a)

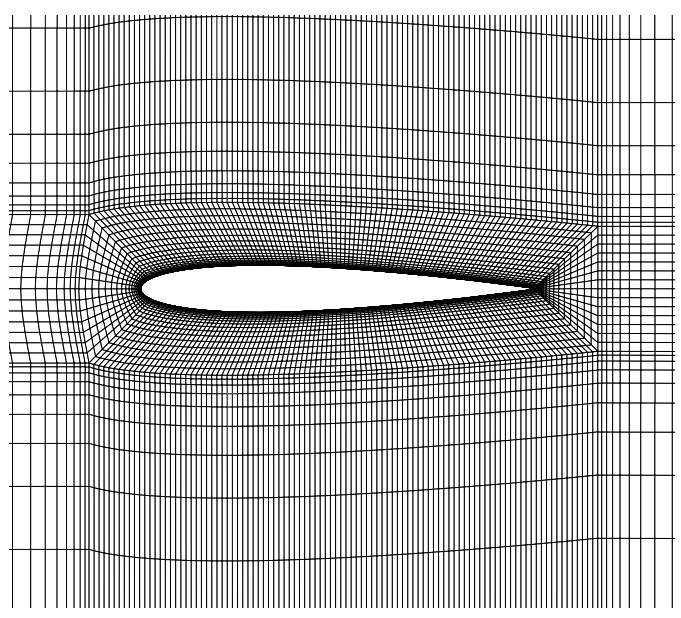

(b)

Figure 1: Spatial convergence study: (a) indicial response of lift coefficient for a step change in angle of attack ( $\Delta \alpha=2.0 \mathrm{deg}, M=0.8)$; (b) medium grid used in this study

For validation of the CFD gust analysis, three test cases are analysed: the sharp-edged gust response in Section 4.1, the step change in angle of attack response in Section 4.2 , and the response to discrete gusts in Section 4.3 .

\subsection{Sharp-edged Gust Response}

Firstly, a time step convergence study was performed. Three values of the time step $(\Delta s=0.01,0.02$, and 0.04$)$ were used. Figure 2(a) shows 


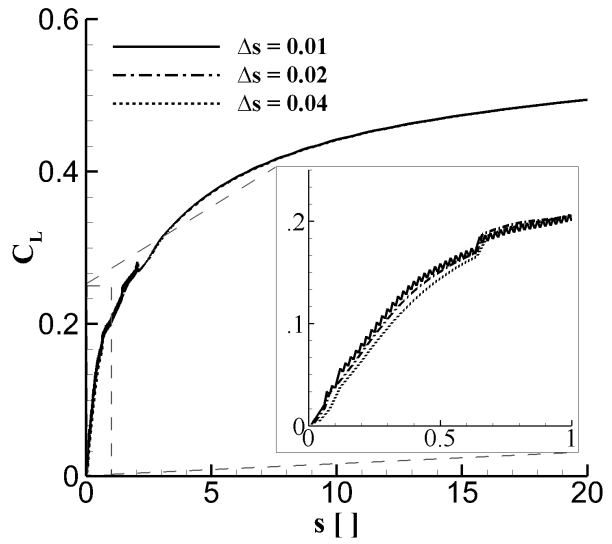

(a)

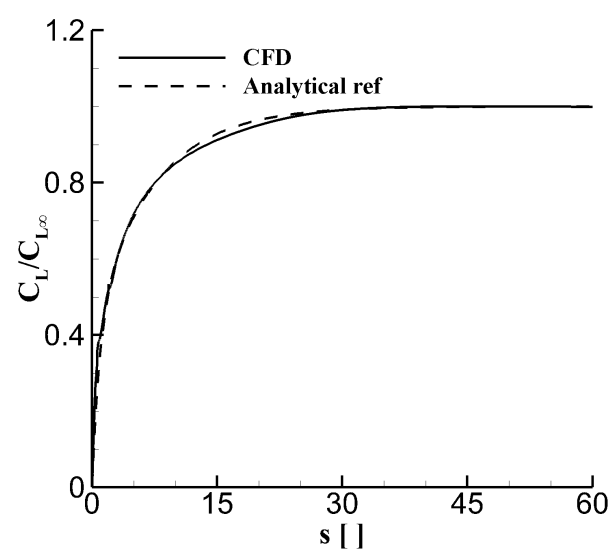

(b)

Figure 2: Indicial response of lift coefficient for a sharp-edged gust normalised by the asymptotic value ( $\hat{w}_{g 0}=0.08, M=0.2$ ): (a) temporal convergence study; (b) analytical reference from Ref. (4) 
penetration into a sharp-edged gust as

$$
\frac{C_{L}}{\hat{w}_{g 0}}=\frac{2 s}{\sqrt{M}} \quad \text { for } 0 \leq s \leq \frac{2 M}{1+M}
$$

Figure 3 shows the computed and analytical results for small times. At lower Mach numbers, results are virtually identical. At the largest Mach number, differences are attributable to compressibility effects not accounted for in the analytical formulation, and the effects of finite thickness of the NACA0012 aerofoil compared to a flat plate. Decreasing the Mach number results in the appearance of oscillations of small entity in the numerical solution as the gust travels over the aerofoil surface. This phenomenon has been reported in a previous study $(10 ; 13)$, and attributed to the convergence process as the gust moves between two adjacent grid points.

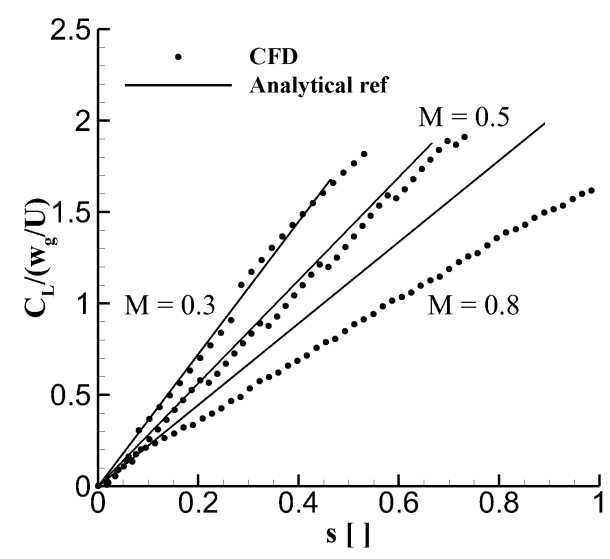

Figure 3: Indicial response of lift coefficient for a sharp-edged gust at small times $\left(\hat{w}_{g 0}=\right.$ 0.08); analytical reference from Ref. (6)

310 Finally, results for a moving sharp-edged gust were also computed. The lift coefficient responses for several values of the advance ratio are shown in 
Figure 4. The gust front moves downstream at a speed $U_{\infty} / \lambda$ relative to the aerofoil: (a) for $\lambda<1$, the gust moves downstream faster than the freestream speed; (b) for $\lambda=1$, the gust has the same speed of the surrounding flow; and (c) for $\lambda>1$, the gust moves downstream slower than the freestream speed. Results are in excellent agreement with those presented in Ref. (10).

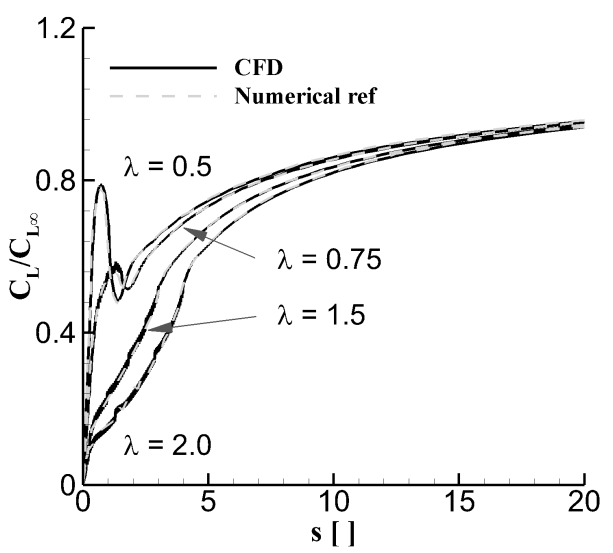

Figure 4: Indicial response of lift coefficient for a moving sharp-edged gust normalised by the asymptotic value $\left(\hat{w}_{g 0}=0.08, M=0.2\right)$; numerical reference from Ref. (10)

\subsection{Step Change in Angle of Attack Response}

The indicial response of the lift coefficient for a step change in angle of attack, $\Delta \alpha=2.0 \mathrm{deg}$, is shown in Figure 5. The indicial response consists of two distinct regions, separated by an intermediate overlapping area. The initial part of the response is representative of the impulsive motion of the body, and of the resulting pressure difference between the upper and lower surfaces where the formation of a compression and expansion wave is observed. The effects of the initial non-circulatory loading are confined within a few chord lengths of the distance travelled. 
333

After the decay of initial transients, the response converges asymptotically to the steady-state value corresponding to the effective angle of attack caused by the step change. The asymptotic value of the circulatory loading can be obtained by linearized quasi-steady theory

$$
\lim _{s \rightarrow \infty} \frac{C_{L}(s)}{\Delta \alpha}=\frac{2 \pi}{\sqrt{1-M^{2}}}
$$

Table 1 summarises analytical and numerical results of the asymptotic values of the indicial response. Increased differences for the highest Mach number are likely because of non-linear compressibility effects captured in the CFD calculations.

Table 1: Asymptotic values of the indicial response of lift coefficient for a step change in angle of attack

\begin{tabular}{ccc}
\hline \hline Mach & Quasi-steady Theory & CFD \\
\hline 0.3 & 6.58 & 6.79 \\
0.5 & 7.25 & 7.49 \\
0.8 & 10.47 & 12.34 \\
\hline \hline
\end{tabular}

An exact analytical expression was obtained by Lomax (6) for a flat plate in a linearised compressible flow. The expression is valid for small times, less than a chord of the distance travelled, and gives

$$
\frac{C_{L}(s)}{\Delta \alpha}=\frac{4}{M}\left(1-\frac{1-M}{2 M} s\right) \quad \text { for } 0 \leq s \leq \frac{2 M}{1+M}
$$

Figure 5(b) shows the comparison between the analytical expression and the numerical results. The qualitative trends are well captured, with some differences becoming more apparent for increasing Mach number. 


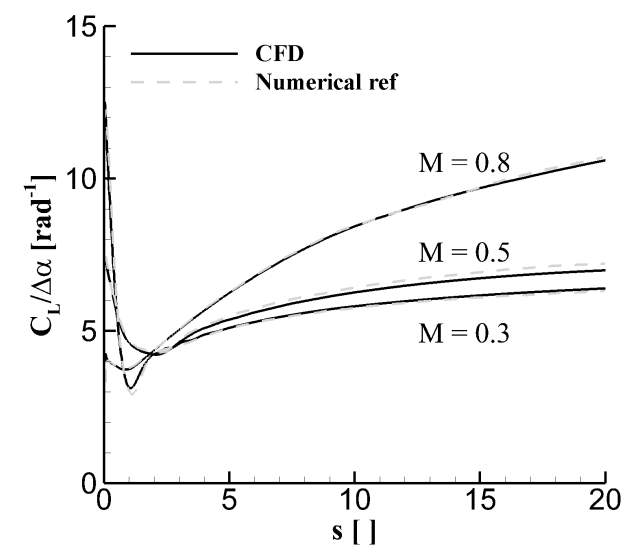

(a) All times; reference from Ref. (26)

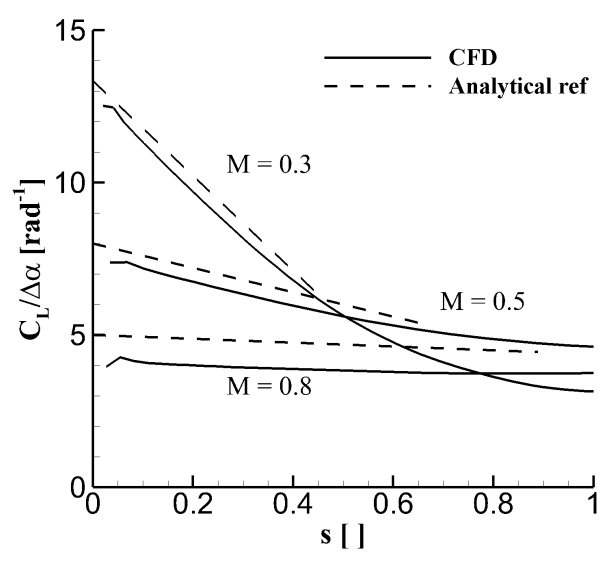

(b) Small times; reference from Ref. (6)

Figure 5: Indicial response of lift coefficient for a step change in angle of attack $(\Delta \alpha=2.0$ $\operatorname{deg})$

\subsection{Discrete Gust Response}

The last validation test case is for a family of discrete gusts. The oneminus-cosine gust has the form

$$
\hat{w}_{g}\left(t, x_{g}\right)=\frac{\hat{w}_{g 0}}{2}\left(1-\cos \left(\frac{2 \pi x_{g}}{H_{g}}\right)\right), \quad \text { for } 0 \leq x_{g} \leq H_{g}
$$

where $x_{g}$ is the position of the aircraft in the spatial description of the gust relative to a convenient fixed frame of reference. Similarly, the sinusoidal gust is given by

$$
\hat{w}_{g}\left(t, x_{g}\right)=\hat{w}_{g 0} \sin \left(\frac{2 \pi x_{g}}{H_{g}}\right), \quad \text { for } 0 \leq x_{g} \leq H_{g}
$$

Figure 6 illustrates the lift coefficient response to both discrete gust shapes. The source of comparison is Ref. (29), and the Mach number and 


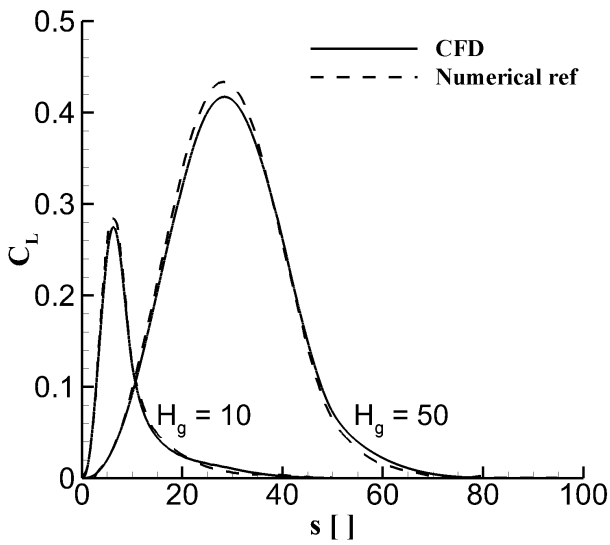

(a) "One-minus-cosine" gust

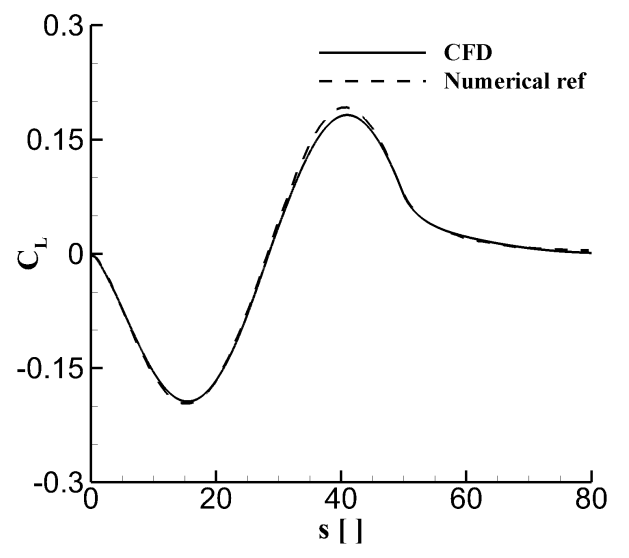

(b) Sinusoidal gust, $H_{g}=50$

Figure 6: Lift coefficient response to discrete gusts $\left(\hat{w}_{g 0}=0.0349, M=0.2\right)$; numerical reference from Ref. (29)

the gust intensity are accordingly set to $M=0.2$ and $\hat{w}_{g 0}=0.0349$, respectively. The response to a one--minus-cosine gust was computed for two gust wavelengths, $H_{g}=10$ and 50 . There is a good agreement for the lift build-up, but small differences were found when the lift coefficient achieves the maximum value. These differences are likely attributed to the different grid used in Ref. (29) from that employed in this work. Qualitatively, similar considerations hold valid for the response to a sinusoidal gust.

\section{Results}

\subsection{Generation of the Reduced Order Model}

The generation of the ROM consists of the following steps.

1. The linearised CFD equations are evaluated for a steady-state flow condition. In this work, the linearisation is carried out for all cases at 
a freestream angle of attack $\alpha_{\infty}=0 \mathrm{deg}$. The validity of the ROM predictions around different mean angles of attack holds valid until second order effects are negligible.

2. A number of POD modes is extracted from an unsteady time--domain analysis. The training data were obtained imposing an impulse function for the angle of attack, $\alpha$, and its time derivative, $\dot{\alpha}$. The impulse function was chosen because it allows exciting the relevant unsteady aerodynamic characteristics. After a sensitivity analysis, 160 POD modes were used given the broad frequency range of the response.

3. A smaller basis of POD modes is formed, extracting a small subset of modes from the original basis and ensuring that $\eta(r)=0.9999$ is achieved. For the aerofoil test case, this was met by selecting 50 POD modes $(r=50)$. Finally, the LTD model is projected onto the basis of 50 POD modes.

Table 2 summarises the computational cost incurred in the generation of the ROM. To provide general insights on the process, CPU times are normalised by the CPU time needed for a steady-state solution to converge 9 orders of magnitude. The breakdown conveys that: (i) the linearisation of the Euler equations is an inexpensive step; (ii) the calculation of the $m=160$ POD snapshots is nearly as costly as a steady-state solution; as already observed in Ref. (30), the reason for this is the slow convergence rate of the GMRES linear solver in the LTD equations; and (iii) the identification of the POD basis consisting of 50 modes requires calculating the singular value decomposition of a large-order snapshot matrix.

The identification of the optimal parameters, $c$ and $\beta$, appearing in the 
Table 2: Computational cost of the ROM generation ( $m=160$ and $r=50$ ); CPU times are normalised by the CPU time needed for a steady-state analysis

\begin{tabular}{lc}
\hline \multicolumn{1}{c}{ ROM Generation Step } & CPU Time \\
\hline Steady-state analysis & 1.00 \\
Linearisation of the time domain equations & 0.11 \\
Generation of $m$ POD modes & 0.89 \\
Extraction of $r$ POD modes and ROM projection & 0.58 \\
\hline \hline
\end{tabular}

ROM gust formulation, Eq. (17), is straightforward. Table (3) reports the values obtained for a sharp-edged gust of intensity $\hat{w}_{g 0}=0.0349$ for several Mach numbers. The values of the parameter $c$ are around unity, and a low dependency on the Mach number was found. This is not unexpected as the parameter relates the intensity of the gust input, $\hat{w}_{g 0}$, to the output, $\alpha_{\mathrm{SE}}$, and is related to steady-state conditions. The transients are ruled by the parameter $\beta$. The increasing trend of the parameter for increasing Mach number reflects the faster dynamics occurring at the higher Mach numbers.

Table 3: Parameters of the gust formulation in the ROM, see Eq. 17.

\begin{tabular}{ccc}
\hline \hline Mach Number & $c$ & $\beta$ \\
\hline 0.3 & 0.9574 & 0.44 \\
0.5 & 0.9613 & 0.75 \\
0.8 & 1.0335 & 0.92 \\
\hline \hline
\end{tabular}

Considering that it is good in practise to initialise an unsteady timedomain analysis from a well-converged steady-state solution, the overall cost of the ROM generation equals 1.72 times the CPU cost of a steady-state run, 
Table 2. Furthermore, the ROM provides the following advantages: (i) once it is generated, the ROM may be employed to parametrically investigate the effects of arbitrary gust profiles on the dynamic response; and (ii) the ROM is of small size, therefore predictions are performed at virtually no extra costs.

\subsection{Step Change in Angle of Attack Response}

Figure 7 shows the indicial response of the lift coefficient for a step change in angle of attack, $\Delta \alpha=2.0 \mathrm{deg}$. Three Mach numbers representative of incompressible, subsonic, and transonic flows are evaluated. The first comparison in Figure 7(a) indicates a good agreement between the nonlinear CFD equations and the LTD counterpart, consisting both of 55,100 degrees of freedom (DoFs). In Figure $7(\mathrm{~b})$, the ROM predictions are superimposed on the LTD solution, revealing an excellent agreement. Here, the ROM consists of 50 DoFs. The comparison provides a first indication on the accuracy of the proposed ROM.

The minimum number of POD modes for accurate predictions is not known a priori, but an estimate of the error can be computed a posteriori. The sensitivity of the ROM results on the number of POD modes was investigated for the $M=0.8$ case of Fig. 7. A number of snapshots (80, 120 and 160) were computed using the LTD solver and then used to form the basis vectors for the POD projection (31). The ROM solutions were computed using the three sets of basis vectors, and compared to the LTD response. Overall, the present technique is in good agreement with the LTD model. For 80 and 120 POD modes, the behaviour in the region connecting the initial part of the impulsive motion with the circulatory part is not well predicted, as shown in Fig. 8. For 160 POD modes, the predicted unsteady 


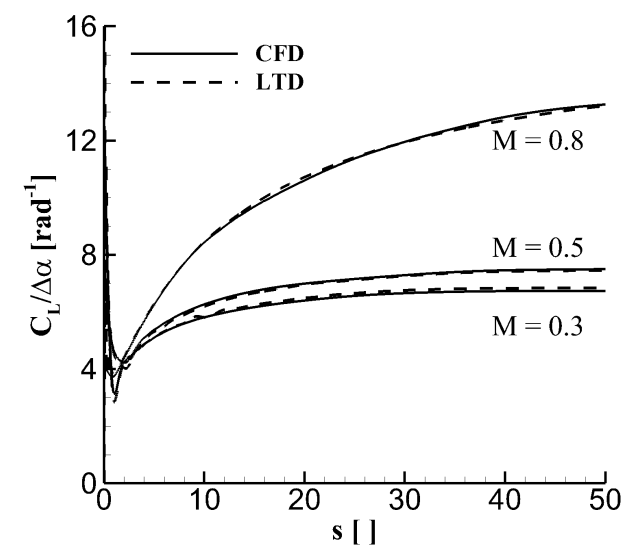

(a)

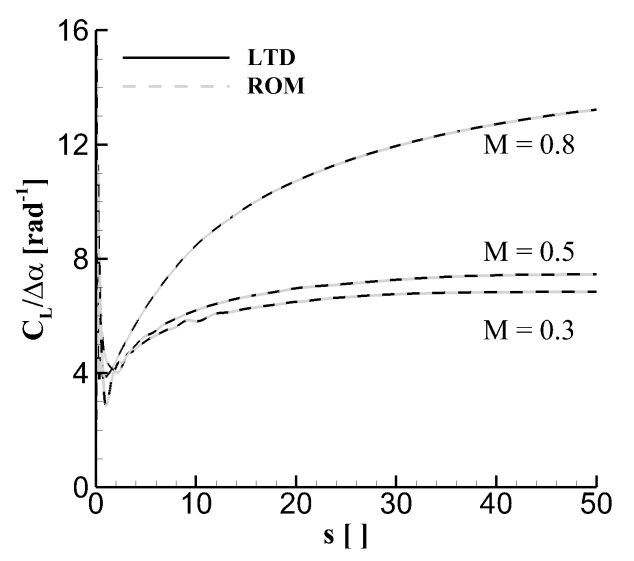

(b)

Figure 7: Indicial response of lift coefficient for a step change in angle of attack ( $\Delta \alpha=2.0$ deg); the CFD and LTD models have 55,100 DoFs, the ROM has 50 DoFs

solution is in excellent agreement with the LTD response. The BT technique was then used to reduce the 160 POD modes into a 50 DoFs ROM that is used for the remainder of this work.

\subsection{Moving Sharp-edged Gust Response}

The case of a moving sharp-edged gust is analysed in Figure 9 for three Mach numbers and three advance ratios. It was found that the ROM reproduces the physics responsible for the decreasing over-shoot of the lift coefficient for increasing Mach number. For the higher $\lambda$, the delay in the lift-up is predicted in the ROM with some discrepancies at the intermediate times compared to the CFD solutions. It was found that the addition of a second exponential in Eq. (17) improves the ROM predictions at small to intermediate times. However, it is worth observing that the present differences between the CFD solver and the ROM are similar, if not smaller, to those 


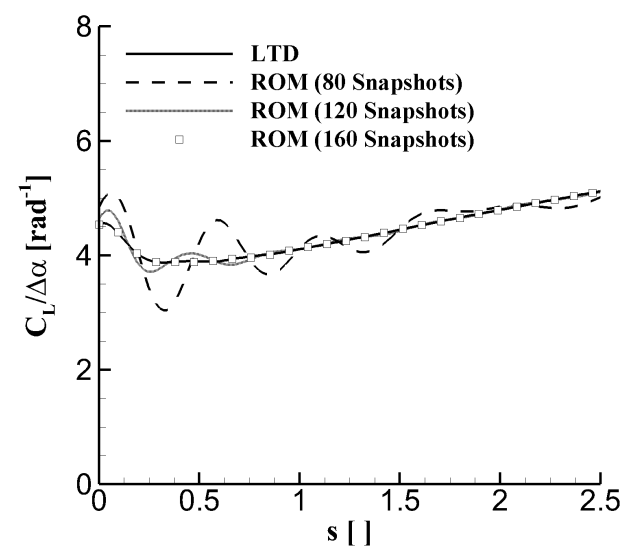

Figure 8: Sensitivity of the ROM on the number of POD modes for the indicial response of lift coefficient for a step change in angle of attack ( $\Delta \alpha=2.0 \mathrm{deg}, M=0.8$ )

observed when using two different CFD codes, as discussed in Ref. (12).

\subsection{Moving Discrete Gust Response}

The final test case is for a family of moving "one-minus-cosine" gusts. The ROM predictions were obtained using the convolution integral proposed in Eq. (19) as an input to the model.

First, Figure 10 compares the response of the lift coefficient at $M=0.8$ and $\hat{w}_{g 0}=0.0349$. Three gust wavelengths $\left(H_{g}=10,20\right.$, and 50) are considered. The ROM parameters were identified from the response to a sharp-edged gust with the same intensity as above, $\hat{w}_{g 0}=0.0349$. The ROM predictions follow closely the FOM response for all gust wavelengths tested. This confirms the adequacy of the ROM gust treatment and the ability to predict the loads response to arbitrary gust shapes.

Then, the ability of the ROM to predict the response to gust intensities $\left(\hat{w}_{g 0}=0.0698\right.$ and 0.1047$)$ other than that used for its generation $\left(\hat{w}_{g 0}=\right.$ 


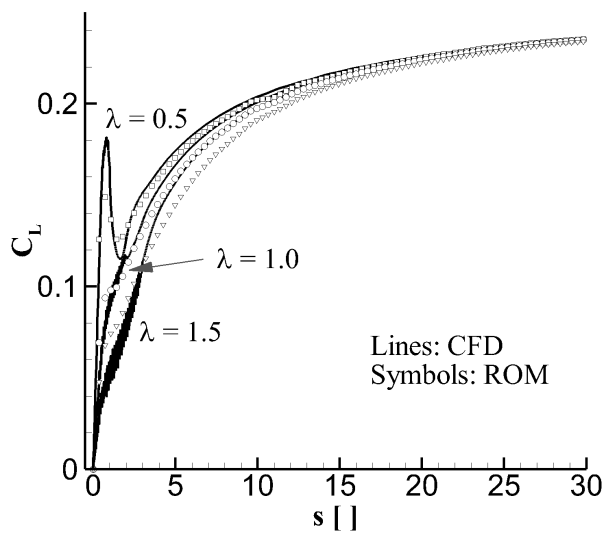

(a) $M=0.3$

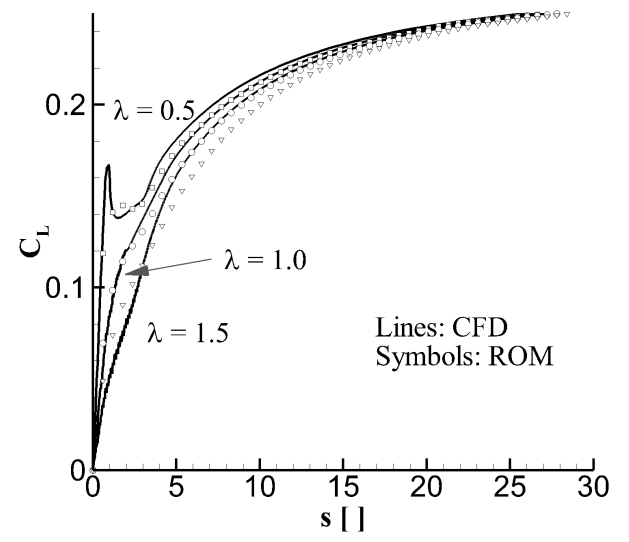

(b) $M=0.5$

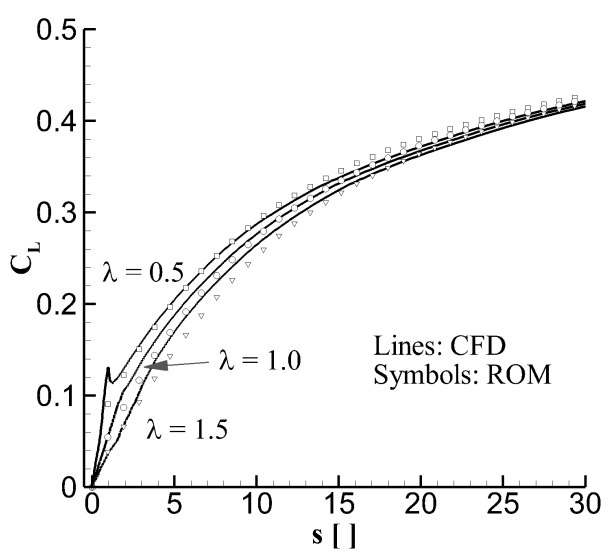

(c) $M=0.8$

Figure 9: Response of lift coefficient for a moving sharp-edged gust $\left(\hat{w}_{g 0}=0.0349\right)$ 


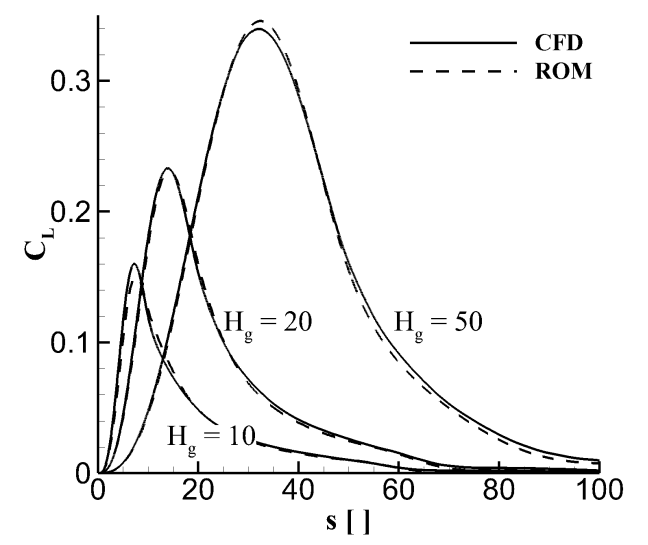

Figure 10: Response of lift coefficient for a "one-minus-cosine" gust $\left(\hat{w}_{g 0}=0.0349\right.$, $M=0.8)$

0.0349) was assessed. In Figure 11, the comparison is carried out at $M=0.8$ for a gust wavelength $H_{g}=20$. To quantify the model accuracy, a fit parameter is introduced

$$
\eta=1-\sqrt{\frac{\sum_{i=1}^{N}\left(C_{L_{i}}-C_{L_{i}}^{\mathrm{ROM}}\right)^{2}}{\sum_{i=1}^{N}\left(C_{L_{i}}\right)^{2}}}
$$

A fit parameter equal unity indicates a perfect match of the ROM results with the CFD results. The fit coefficient is reported for the three gust intensities in Table 4. It is not unexpected that the error increases for increasing gust intensity because the flow field characteristics depart more markedly from the reference steady-state solution. For example, Figure 12 compares the pressure coefficient distribution at $M=0.8$ for two flow conditions. The first is for the steady solution at $\alpha=0 \mathrm{deg}$, which was used to generate the ROM, featuring a symmetric shock wave. The second condition depicts the instantaneous flow solution when the lift coefficient reaches the highest peak 
for $\hat{w}_{g 0}=0.1047$ in Figure 11. The shock disappears from the lower surface and becomes stronger on the upper surface.

Similarly, a study was conducted to assess the validity of the ROM around mean angles of attack different from the one used for its generation, $\alpha=0.0$ deg. For the transonic case, $M=0.8$, with a "one-minus-cosine" gust of intensity $\hat{w}_{g 0}=0.0349$ in Figure 11 , second order effects start to become significant above 2 deg mean angle of attack.

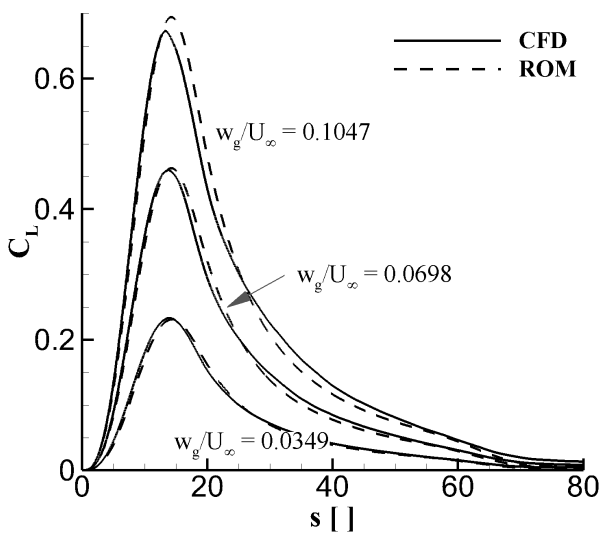

Figure 11: Response of lift coefficient for a "one-minus-cosine" gust $\left(H_{g}=20, M=0.8\right)$

Table 4: Fit parameter of the lift coefficient for a "one-minus-cosine" gust $\left(H_{g}=20\right.$, $M=0.8$ )

\begin{tabular}{cc}
\hline \hline$\hat{w}_{g}$ & $\eta$ \\
\hline 0.0349 & 0.979 \\
0.0698 & 0.961 \\
0.1047 & 0.940 \\
\hline \hline
\end{tabular}




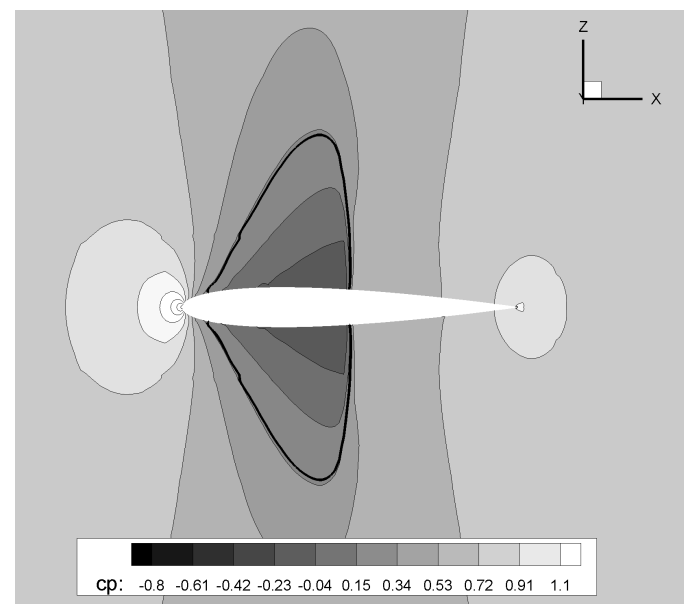

(a) Steady, $\alpha=0$ deg

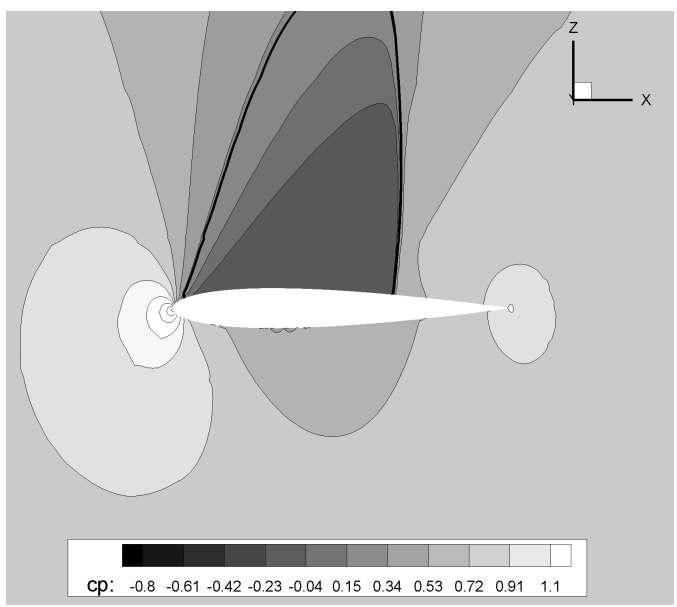

(b) Instantaneous, $\alpha \approx 6 \mathrm{deg}$

Figure 12: Pressure coefficient distribution during the response to a "one-minus-cosine" gust ( $\hat{w}_{g 0}=0.1047, H_{g}=20, M=0.8$ ): (a) steady solution at $\alpha=0 \mathrm{deg}$, (b) snapshot corresponding to maximum $C_{L}$ for $\hat{w}_{g 0}=0.1047$ in Figure 11 the solid thick line indicates $M=1.0$ 
Finally, the case of a moving "one-minus-cosine" gust is analysed in Figure 13 at $M=0.8$ and with gust parameters $\hat{w}_{g 0}=0.0349$ and $H_{g}=20$. An excellent agreement is observed in all cases. For increasing advance ratio, the horizontal speed of the gust in the downstream direction decreases. As the total time of the gust-aerofoil interaction increases, so does the effect of the gust on the aerodynamic loads.

Results in Figure 13 illustrate the impact of the advance ratio on the aerodynamic loads around a rigid configuration. For a gust of given intensity and wavelength, as in the test case herein considered, the action of the advance ratio translates into an apparent change of both intensity and wavelength. This aspect of gusts, which has a low to null coverage in the open literature, is more critical for low speed flight, as typical of high altitude long endurance aircraft. To date, however, certification requirements for structural sizing do not include any relevant information in this regard. For a flexible structure, the horizontal velocity component of the gust is likely to affect the aeroelastic response to a higher degree compared to a rigid configuration. An initial work in this direction is given in Ref. (22).

\subsection{Computational Efficiency}

The computational cost of the ROM generation was addressed in Section 5.1, while this Section is concerned with the computational efficiency of time integrating the ROM equations. The settings of the unsteady timedomain analysis (time step size, final simulation time) are identical between the CFD and the ROM. Two cases are evaluated: (i) the response for the moving sharp-edge gust shown in Figure 9; and (ii) the response for the "one-minus-cosine" gust of Figure 10. Table 5 summarises the computa- 


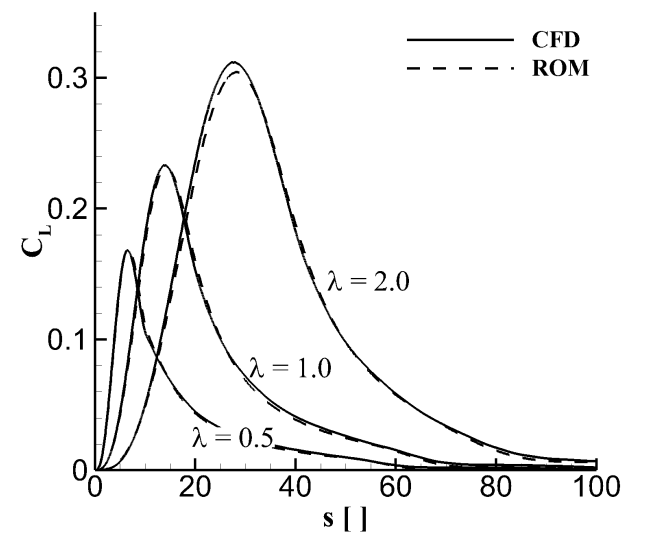

Figure 13: Response of lift coefficient for a moving "one-minus-cosine" gust $\left(\hat{w}_{g 0}=\right.$ $\left.0.0349, H_{g}=20, M=0.8\right)$

Table 5: Computational cost comparison between CFD and ROM for various gust shapes

tional efficiency of the ROM compared to the CFD solver. Values are normalised by the CPU time of the ROM to complete the unsteady analysis. It was found that the computational efficiency of the ROM maintains nearly constant for both cases, with a speed-up of about 300 times. For reference, the ROM calculations were performed in about one second on a standard computer.

\begin{tabular}{lcc}
\hline \hline & $\begin{array}{c}\text { Sharp-edged gust } \\
\text { (see Figure }\end{array}$ & $\begin{array}{r}\text { "One-minus-cosine" gust } \\
\text { (see Figure }\end{array}$ \\
\hline CFD time integration, Eq. (3) & 310.0 & 296.8 \\
ROM time integration, Eq. (16) & 1.0 & 1.0 \\
\hline \hline
\end{tabular}




\section{Conclusions}

The novelty of the proposed work is on the gust treatment within a reduced order model, which is based on a proper orthogonal decomposition representation of the linearised time-domain equations. The approach builds on two requirements, speed and simplicity, and combines within the same framework analytical and numerical evaluations. The analytical component is inspired by the Küssner function which models the lift built-up for a sharp-edged gust through exponential functions. Unknown parameters of the analytical component are then identified from an appropriate training response. To demonstrate the application of the new methodology, a model problem for the inviscid flow around a standard aerofoil section is thoroughly evaluated.

First, an extensive validation of the computational fluid dynamics solver for gust loads is carried out using available data in the open literature. Then, the reduced order model is demonstrated for a variety of test cases, including the step change in angle of attack, moving sharp-edged gust, and moving discrete gusts both in the incompressible and compressible flow regimes. Three assessments were performed to draw conclusions on the reduced order model: (i) cost of the reduced order model generation; (ii) accuracy of the reduced order model predictions; and (iii) computational efficiency of the reduced order model. The cost of the reduced order model generation is critical, but shall be balanced against the accuracy of predictions. It was found that the model generation was performed at the equivalent cost of less than two steady-state analyses, and that the most expensive step was identified to be the generation of the proper orthogonal modes. The predictions from the 
reduced order model were found in good to excellent agreement with the computational fluid dynamics simulations for all gust shapes and flow conditions. Finally, the computational efficiency of time integrating the reduced order model compared to the computational fluid dynamics equations was assessed. Employing the same numerical settings, the reduced order model achieved a speed-up of about 300 times consistently for two test cases.

In conclusion, the proposed methodology to introduce gust effects in the reduced order model is deemed accurate and relatively inexpensive, opening up a potential application in the parametric search of the worst-case loads. Whereas this work has focused on the methodology demonstrated on a model problem, the application to three-dimensional aeroelastic problems has already been performed successfully and will be reported separately.

Lastly, a consideration on the impact of the advance ratio on the aerodynamic loads is worth mentioning. For a gust of given intensity and wavelength, the action of the advance ratio translates into an apparent change of both intensity and wavelength. This aspect of gust loads analysis seems absent in the literature and, most importantly, is not included in current certification requirements for structural sizing. Certainly, this becomes more critical for low speed flight, as typical of high altitude long endurance aircraft and it is expected to have a large impact on flexible structures.

\section{Acknowledgements}

This work was supported by the National Program on Key Research Projects (No: MJ-2015-F-010), the National Natural Science Foundation of China (Nos. 11272005, 11472206, 11672225 and 11511130053), and the 
Basic Research foundations for the Central Universities (2014XJJ0126). Andrea Da Ronch also acknowledges the financial contribution from the Royal Academy of Engineering (NCRP/1415/51).

\section{References}

[1] Bensch, L., Henrichfreise, H., Jusseit, J., and Merz, L., "Method for reconstructing gusts and structural loads at aircraft, in particular passenger aircraft," June 26 2007, US Patent 8,209,159.

[2] Theodorsen, T., "General Theory of Aerodynamic Instability and the Mechanism of Flutter," NACA Report No. 496, 1935.

[3] Jones, R. T., "The Unsteady Lift of a Wing of Finite Aspect Ratio," NACA Report No. 681, 1940.

[4] Leishman, J. G., "Unsteady Lift of a Flapped Airfoil by Indicial Concepts," Journal of Aircraft, Vol. 31, No. 2, 1994, pp. 288-297.

[5] Miles, J. W., "The Aerodynamic Force on an Airfoil in a Moving Gust," Journal of Aerospace Sciences, Vol. 23, No. 11, 1956, pp. 1044-1050.

[6] Lomax, H., "Indicial Aerodynamics," AGARD Manual of Aeroelasticity, Part II, Chapter 6, 1960.

[7] Lighthill, M. J., "Oscillating Airfoils at High Mach Number," Journal of Aeronautical Sciences, Vol. 20, No. 6, 1953, pp. 402-406.

[8] Parameswaran, V. and Baeder, J. D., "Indicial Aerodynamics in Compressible Flow-Direct Computational Fluid Dynamic Calculations," Journal of Aircraft, Vol. 34, No. 1, 1997, pp. 131-133. 
[9] Ghoreyshi, M., Cummings, R. M., Da Ronch, A., and Badcock, K. J., "Transonic Aerodynamic Loads Modeling of X-31 Aircraft Pitching Motions," AIAA Journal, Vol. 51, No. 10, 2013, pp. 2447-2464, doi: 10.2514/1.J052309.

[10] Da Ronch, A., On the Calculation of Dynamic Derivatives Using Computational Fluid Dynamics, Ph.d. thesis, School of Engineering, University of Liverpool, U.K., 2012.

[11] Righi, M., Berci, M., and Koch, J., "Subsonic Indicial Aerodynamics for Unsteady Loads Calculation via Numerical and Analytical Methods: a Preliminary Assessment," AIAA Aviation, AIAA-2015-3170, 22-26 June 2015, doi: 10.2514/6.2015-3170.

[12] Righi, M., Berci, M., Franciolini, M., Da Ronch, A., and Kharlamov, D., "Subsonic Indicial Aerodynamics for the Unsteady Loads of Trapezoidal Wings," AIAA Aviation, AIAA-2016-3688, 13-17 June 2016.

[13] Raveh, D. E., "CFD-Based Models of Aerodynamic Gust Response," Journal of Aircraft, Vol. 44, No. 3, 2007, pp. 888-897, doi: $10.2514 / 1.25498$.

[14] Zhang, W., Ye, Z., Yang, Q., and Shi, A., "Gust Response Analysis Using CFD-Based Reduced Order Models," 47th AIAA Aerospace Sciences Meeting, AIAA-2009-0895, 05-08 January 2009, doi: 10.2514/6.2009895.

[15] Bisplinghoff, R. L., Ashley, H., and Halfman, R. L., Aeroelasticity, Addison-Wesley, 1955. 
[16] Bartels, R., "Developing an Accurate CFD Based Gust Model for the Truss Braced Wing Aircraft," 31st AIAA Applied Aerodynamics Conference, Fluid Dynamics and Co-located Conferences, AIAA-2013-3044, 24-27 June 2013, doi: 10.2514/6.2013-3044.

[17] Chen, G., Sun, J., and Li, Y. M., "Active flutter suppression control law design method based on balanced proper orthogonal decomposition reduced order model," Nonlinear Dynamics, Vol. 70, No. 1, 2012, pp. 112, doi: 10.1007/s11071-012-0392-4.

[18] Chen, G., Wang, X., and Li, Y., "A reduced-order-model-based multiple-in multiple-out gust alleviation control law design method in transonic flow," Science China Technological Sciences, Vol. 57, No. 2, 2014, pp. 368-378, doi: 10.1007/s11431-013-5416-x.

[19] Da Ronch, A., Badcock, K. J., Wang, Y., Wynn, A., and Palacios, R. N., "Nonlinear Model Reduction for Flexible Aircraft Control Design," AIAA Atmospheric Flight Mechanics Conference, AIAA-20124404, 13-16 August 2012, doi: 10.2514/6.2012-4404.

[20] Da Ronch, A., Tantaroudas, N. D., Timme, S., and Badcock, K. J., "Model Reduction for Linear and Nonlinear Gust Loads Analysis," 54th AIAA/ASME/ASCE/AHS/ASC Structures, Structural Dynamics, and Materials Conference, AIAA-2013-1492, 08-11 April 2013, doi: $10.2514 / 6.2013-1492$.

[21] Timme, S., Badcock, K. J., and Da Ronch, A., "Linear Reduced Order Modelling for Gust Response Analysis using the DLR-TAU Code," In- 
ternational Forum on Aeroelasticity and Structural Dynamics (IFASD), IFASD-2013-36A, 24-27 June 2013.

[22] Qiang, Z., Chen, G., Li, Y., and Da Ronch, A., "Transonic Aeroelastic Moving Gust Responses and Alleviation based on CFD," AIAA Aviation, AIAA-2016-3837, 13-17 June 2016, doi: 10.2514/6.2016-3837.

[23] Van Leer, B., "Towards the ultimate conservative difference scheme. V. A second-order sequel to Godunov's method," Journal of Computational Physics, Vol. 32, No. 1, 1979, pp. 101-136, doi: 10.2514/1.25498.

[24] Chen, G., Li, Y., and Yan, G., "A nonlinear POD reduced order model for limit cycle oscillation prediction," Science China Physics, Mechanics and Astronomy, Vol. 53, No. 7, 2010, pp. 1325-1332, doi: 10.1007/s11433-010-4013-2.

[25] Zhou, Q., Li, D., Da Ronch, A., Chen, G., and Li, Y., "Computational fluid dynamics-based transonic flutter suppression with control delay," Journal of Fluids and Structures, Vol. 66, 2016, pp. 183-206, doi: 10.1016/j.jfluidstructs.2016.07.002.

[26] Singh, R. and Baeder, J. D., "Direct Calculation of Three-Dimensional Indicial Lift Response Using Computational Fluid Dynamics," Journal of Aircraft, Vol. 34, No. 4, 1997, pp. 465-471.

[27] Lesoinne, M., Sarkis, M., Hetmaniuk, U., and Farhat, C., "A linearized method for the frequency analysis of three-dimensional fluid/structure interaction problems in all flow regimes," Computer Methods in Applied 
Mechanics and Engineering, Vol. 190, No. 24-25, 2001, pp. 3121-3146, doi: 10.1016/S0045-7825(00)00385-6.

[28] Badcock, K. J., Timme, S., Marques, S., Khodaparast, H., Prandina, M., Mottershead, J., Swift, A., Da Ronch, A., and Woodgate, M., “Transonic aeroelastic simulation for envelope searches and uncertainty analysis," Progress in Aerospace Sciences, Vol. 47, No. 5, 2011, pp. 392-423, doi: 10.1016/j.paerosci.2011.05.002.

[29] Zaide, A. and Raveh, D. E., "Numerical Simulation and Reduced-Order Modeling of Airfoil Gust Response," AIAA Journal, Vol. 44, No. 8, 2006, pp. 1826-1834, doi: 10.2514/1.16995.

[30] Amsallem, D., Interpolation on manifolds of CFD-based fluid and finite element-based structural reduced-order models for on-line aeroelastic predictions, Ph.d. thesis, Department of Aeronautics and Astronautics, Stanford University, 2010.

[31] Ekici, K. and Hall, K. C., "Fast Estimation of Unsteady Flows in Turbomachinery at Multiple Interblade Phase Angles," AIAA Journal, Vol. 44, No. 9, 2006, pp. 2136-2142, doi: 10.2514/1.23288. 